\title{
Asymmetric Modulation of Cytosolic cAMP Activity Induces Growth Cone Turning
}

\author{
Ann M. Lohof, Mark Quillan, ${ }^{a}$ Yang Dan, and Mu-ming Poo \\ Department of Biological Sciences, Columbia University, New York, New York 10027
}

\begin{abstract}
The possible role of cyclic nucleotides as second messengers mediating growth cone turning was studied by producing an asymmetric distribution of cyclic nucleotides across the growth cone. A repetitive pulse application method was developed to produce microscopic chemical gradients near the growth cone of embryonic Xenopus neurons in cell culture. When picoliters of a solution containing $20 \mathrm{~mm}$ dibutyryl cAMP (dB-cAMP), a membrane-permeable analog of CAMP, were repetitively ejected from a micropipette near the growth cone, neurite growth was consistently directed toward the pipette. Theoretical analysis of the diffusion gradient showed that the neurite is capable of detecting a $10 \%$ difference in dB-cAMP concentration across the growth cone. Similar responses were also observed using gradients of the phosphodiesterase inhibitor isobutylmethylxanthine, or of forskolin, which activates adenylate cyclase. Dibutyryl cGMP, however, produced no significant turning. These results suggest that a cytoplasmic gradient of CAMP across the growth cone is sufficient to initiate its turning response, and that CAMP in the growth cone could serve as a second messenger in mediating the action of extracellular guidance cues.
\end{abstract}

Formation of specific nerve connections in the developing nervous system requires that the nerve growth cone make appropriate orientation responses during its pathfinding. Turning of the growth cone can be induced by a number of physical and chemical factors in the extracellular environment, including chemotactic substances, mechanical barriers, substratum adhesiveness, and selective affinity for nearby cells (reviewed in Letourneau, 1985; Bray and Hollenbeck, 1988). These external factors, despite differences in their initial actions on the growth cone, may trigger a common set of intracellular events involving cytoplasmic second messengers. A gradient of cytoplasmic second messenger may in turn signal the preferential incorporation of new plasma membrane material and asymmetric cytoskeleton reorganization at the growth cone that are required for the appropriate orientation of the neurite.

cAMP is a common second messenger involved in many cellular responses to extracellular agents. Alteration of cAMP activity has been shown to increase survival and neurite out-

\footnotetext{
Received Aug. 26, 1991; revised Nov. 1, 1991; accepted Nov. 6, 1991.

This work was supported by grants from U.S. National Institutes of Health (NS-22764) and National Science Foundation (BNS-13306, BNS-12398). We thank N. Tabti and C. Scheffey for comments on the manuscript and Z. Guan for excellent technical assistance.

Correspondence should be addressed to Dr. Mu-ming Poo at the above address.

a Present address: Department of Pharmacology, Yale University School of Medicine, New Haven, CT 06510.

Copyrignt (C) 1992 Society for Neuroscience $0270-6474 / 92 / 121253-09 \$ 05.00 / 0$
}

growth in some cases (Rydel and Greene, 1988; Tsuda et al., 1989; Sano et al., 1990) but to inhibit outgrowth and growth cone motility in other situations (Greene et al., 1986; Lankford et al., 1988; Mattson et al., 1988; Bixby, 1989). Elevation of the cytosolic cAMP concentration by application of forskolin promotes the forward movement of organelles into the lamellar region of the Aplysia growth cone (Forscher et al., 1987), which may be a prelude to the new membrane incorporation at the growth cone. Since a similar effect is produced by cytochalasins (Forscher and Smith, 1988), cAMP may act by modifying the actin network in the growth cone. Elevated cAMP activity could also affect microtubule polymerization and interaction with other cytoskeletal elements that are essential for growth cone motility and neurite extension.

In the present study, we aimed to determine whether a gradient of cytosolic cAMP in the growth cone could lead to neurite reorientation in the direction of the gradient. In a previous study on the turning response of chick dorsal root neurites toward NGF, Gundersen and Barrett (1980) reported that $100 \%$ of these neurites grew toward sources of mono- and dibutyryl cAMP and cGMP. Only 10-20 neurons were examined under each condition, and the growth cone responses were scored qualitatively. In order to substantiate this important finding with quantitative data, we decided to examine the response of Xenopus spinal neurites in defined gradients of chemicals that perturb asymmetrically the cytosolic cyclic nucleotide levels. A new method of repetitive pulse application was developed in order to create reproducible chemical gradients. Assuming that an extracellular gradient of membrane-permeable agent produced a corresponding cytosolic gradient of cAMP activity, our results indicate that a cytoplasmic gradient of cAMP is sufficient to initiate growth cone turning. However, we found no evidence for turning toward cGMP analogs. In addition to specific questions addressed in this study, the method described here for producing chemical gradients by pulsatile application may also prove to be useful for testing the chemotactic responses of growth cones to putative neurotropic substances.

\section{Materials and Methods}

Culture methods and chemicals. Cultures were prepared according to procedures reported previously (Spitzer and Lamborghini, 1976; Anderson et al., 1977; Tabti and Poo, 1991). Briefly, the neural tube tissue from 1-d-old embryos (stage 20-24; Nieuwkoop and Faber, 1967) was dissociated in $\mathrm{Ca}^{2+} / \mathrm{Mg}^{2+}$-free Ringer's solution supplemented with EDTA, plated on clean glass coverslips, and incubated at room temperature $\left(20-22^{\circ} \mathrm{C}\right)$ for $6-12 \mathrm{hr}$ prior to the expcriment. The culture medium consisted of $50 \%(\mathrm{v} / \mathrm{v})$ Leibovitz's medium (GIBCO), $2 \%(\mathrm{v} /$ v) fetal calf serum (GIBCO), and $48 \%$ (v/v) Ringer's solution (115 mM $\mathrm{NaCl}, 2$ mM CaCl, $2.5 \mathrm{~mm} \mathrm{KCl}$, and $10 \mathrm{~mm}$ HEPES, pH 7.3). Dibutyryl cAMP (dB-cAMP), dibutyryl cGMP (dB-cGMP), and isobutylmethyl- 
Figure 1. Diffusion profile of Trypan blue dye at various times after a single pulse ejection of concentrated dye solution from the tip of a micropipette. The duration of the pulse was $10 \mathrm{msec}$. Scale bar, $30 \mu \mathrm{m}$.
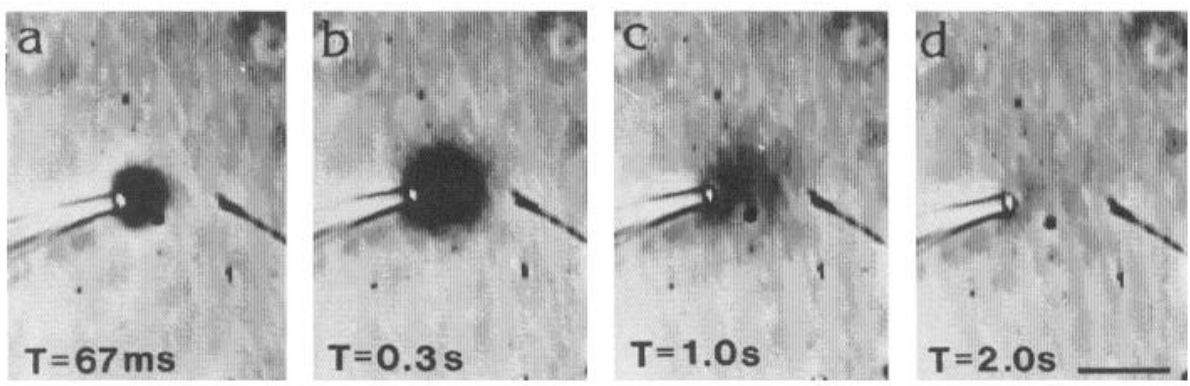

xanthine (IBMX) were obtained from Sigma. Forskolin (7 $\beta$-desacetyl$7 \beta$-[ $\gamma$-( $N$-methylpiperazino)-butyryl] $)$ or L 858051 , a derivative of forskolin reported to have increased stability and water solubility, was obtained from Calbiochem. All test substances were dissolved in serumfree culture medium.

Production of microscopic gradients. In preliminary studies, we employed a previously reported method (Gunderson and Barrett, 1980) in which a gradient near the growth cone was produced by a steady ejection of chemical solution from a micropipette against a perfusion flow of medium across the entire culture. However, due to unpredictable turbulence caused by the two colliding flows, this method yielded irreproducible gradients in our hands. To avoid the introduction of medium flow, we developed the pulsatile ejection method used in the present study. Micropipettes used in pulsatile ejection were pulled with a twostage puller designed for making patch-clamp electrodes (Narashige PP83 ) and heat polished to reduce the inner diameter of the tip to between 1-2 $\mu \mathrm{m}$ on a microforge (Narashige MF-83). The pipettes were filled with test solution and connected to an electrically gated pressure application system (Picospritzer, General Valve Co.). Positive pressure of 2-3 psi was applied to the pipette with defined frequency and duration using a pulse generator (Grass SD9). The average volume of the ejected solution per pressure pulse was determined by measuring the volume of droplets ejected into oil for standard pipettes of $1 \mu \mathrm{m}$ or $2 \mu \mathrm{m}$ inner tip diameter. Because of the small volume of each pulse, the average volume per pulse was estimated from the total volume of 30-50 pulses. For the condition of $1 \mu \mathrm{m}$ tip diameter, duration $4 \mathrm{msec}$, and pressure
Figure 2. Analysis of chemical gradients produced by focal pulsatile application method. $a$, Concentration profile at various times after the pressure ejection of a single pulse (duration $20 \mathrm{msec}$ ) of fluorescent dye, fluorescein-conjugated dextran (Sigma, MW 9000). Symbols represent normalized values of fluorescence intensities measured at various distances from the ejection site at $0.3 \sec (\nabla), 0.9 \sec (\bullet)$, and $1.7 \mathrm{sec}(O)$ after the pulse ejection. Same normalization parameters were used to normalize all three sets of data. Solid lines represent theoretical predicted concentration profile at $0.3,0.9$, and $1.7 \mathrm{sec}$, respectively, assuming a diffusion coefficient of $2.0 \times 10^{-6} \mathrm{~cm}^{2} /$ sec for the dextran. All three sets of data were normalized by a single scaling factor to fit theoretical curves given by Equation 1 in Materials and Methods. $b$. Calculated concentration gradients of dB-cAMP at distances 50, 100, and 200 $\mu \mathrm{m}$ from the micropipette after repetitive pulse applications of $0.3 \mathrm{pl}$ of 20 $\mathrm{mm}$ solution at a pulse frequency of 0.5 $\mathrm{Hz}$ and duration of $4 \mathrm{msec}$, using Equation 2. Diffusion coefficient of dB-cAMP used in the calculation was $5.2 \times 10^{-6}$ $\mathrm{cm}^{2} / \mathrm{sec}$ (Weast, 1984). c Calculated concentration with time after the onset of repetitive pulses of $0.5,1$, and $2 \mathrm{~Hz}$ at a distance of $100 \mu \mathrm{m}$ from the source. Pulse parameters, except the frequency, were the same as in $b . d$, Calculated concentration gradients of the chemical with time at a distance of $100 \mu \mathrm{m}$ from the source for the same conditions as those in $c$.
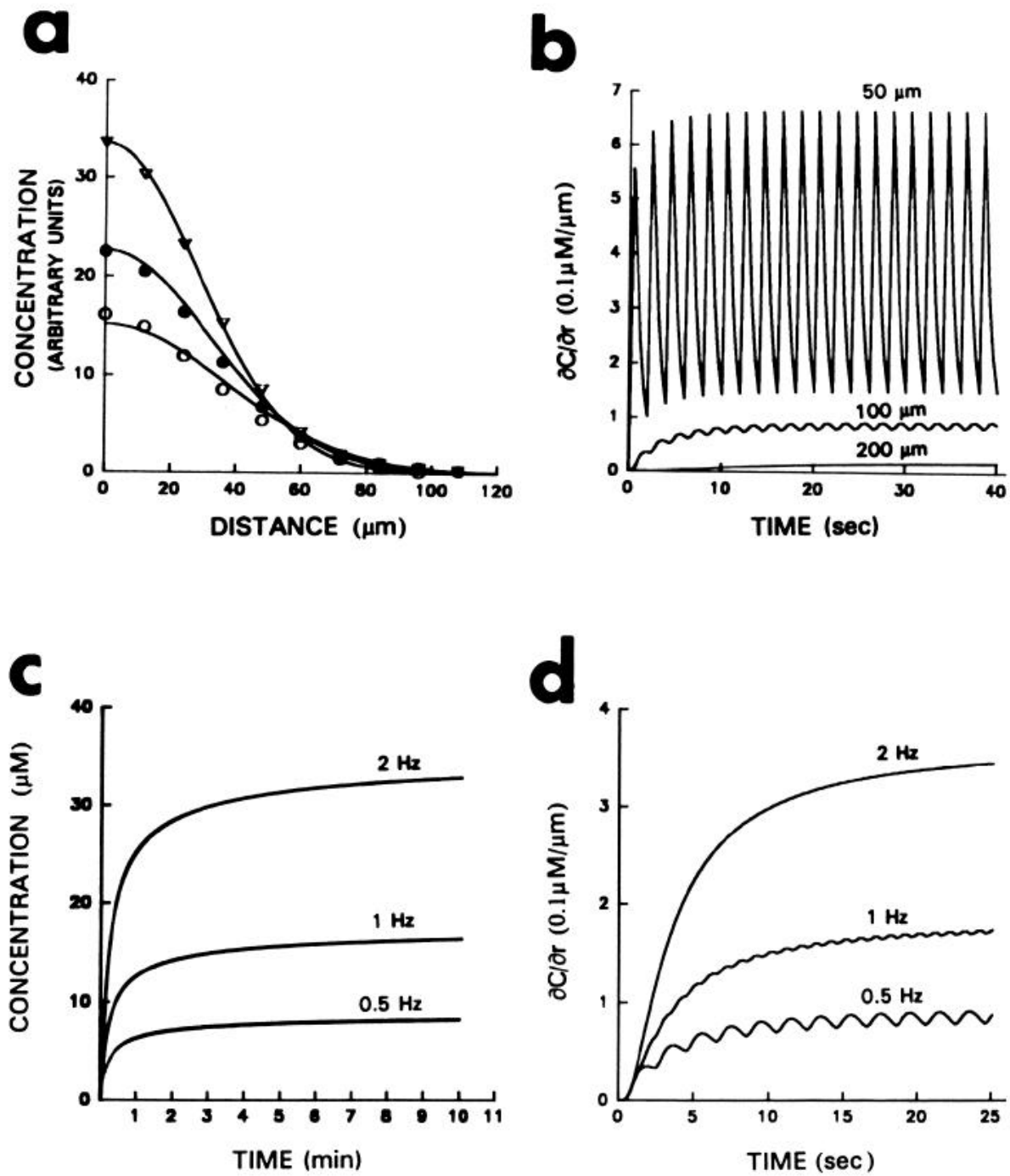

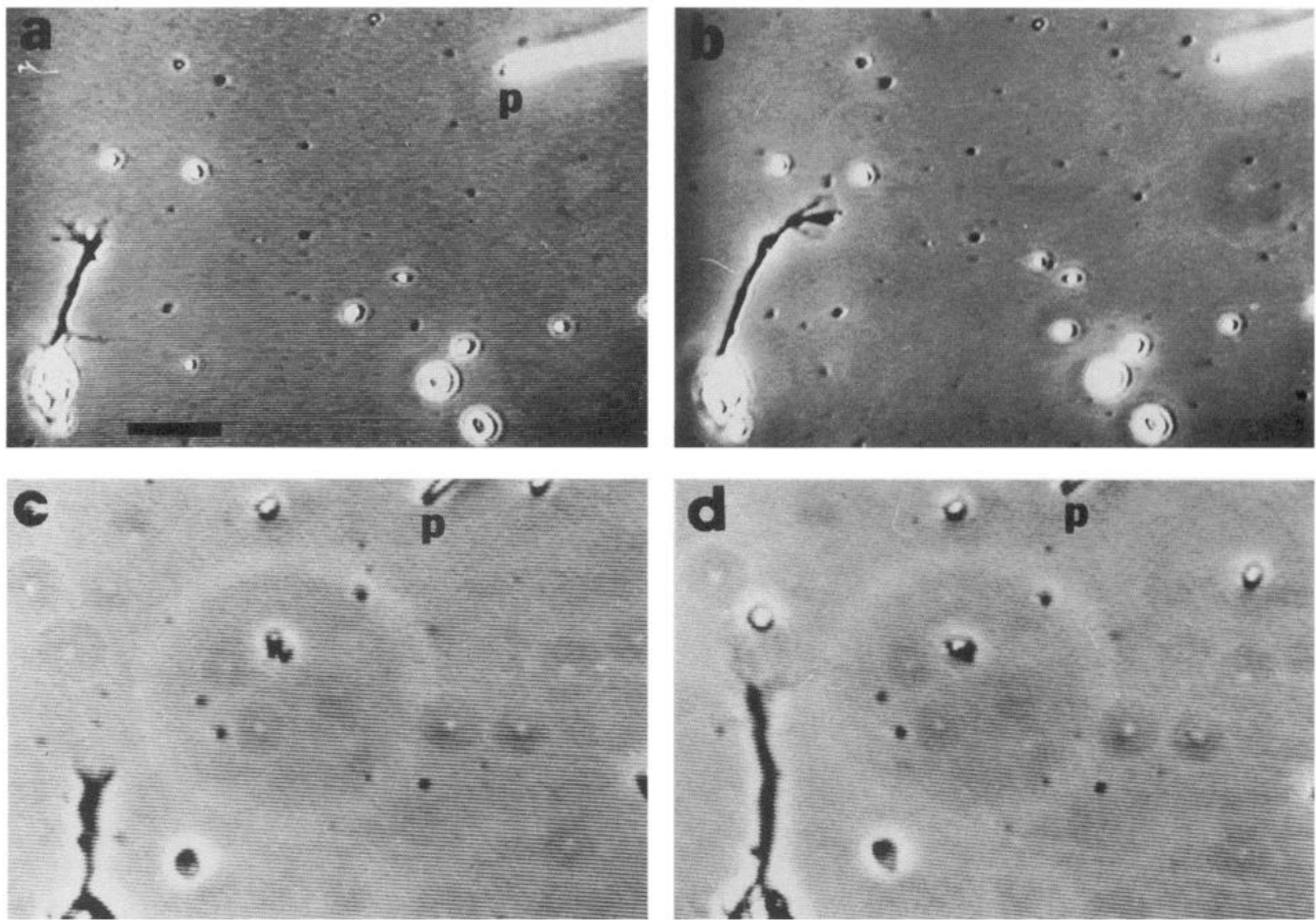

Figure 3. Turning response of Xenopus spinal neurons in the presence of chemical gradients. Representative phase-contrast micrographs of isolated neurons before $(a, c)$ and $2 \mathrm{hr}$ after $(b, d)$ the onset of pulsatile application of chemicals from a micropipette $(p)$ positioned at a distance of 100 $\mu \mathrm{m}$ from the center of the growth cone and an angle of $45^{\circ}$ from the direction of neurite extension. $a$ and $b$, The pipette contained 20 mM dBcAMP. Pulse frequency, $0.5 \mathrm{~Hz}$; pulse duration, $4 \mathrm{msec}$, volume ejected per pulse, $0.3 \mathrm{pl} . c$ and $d$, Same conditions as $a$ and $b$ except that $\mathrm{dB}$ cAMP was replaced by sucrose. Scale bar, $20 \mu \mathrm{m}$.

level $3 \mathrm{psi}$, we obtained an average pulse volume of $0.30 \mathrm{pl}(N=6)$. For $2 \mu \mathrm{m}$ tip diameter, $10 \mathrm{msec}$, and $3 \mathrm{psi}$, the average volume was $0.65 \mathrm{pl}(N=5)$. To illustrate the diffusion profile, Figure 1 shows the dye distribution at various times after a pulse of concentrated dye solution was ejected from a micropipette of a large tip opening.

Theoretical analysis of the gradient. The concentration $(C)$ and the concentration gradient $(\partial C / \partial r)$ after repetitive ejection of the chemical from the micropipette were computed by summing the concentration profile from individual pulses. Assuming that diffusion of the chemical from each ejected pulse behaves as diffusion from a point source, the concentration profile of the chemical after a single ejection is described by the following formula:

$$
c(r, t)=\left[A / 4(\pi D t)^{3 / 2}\right] \exp \left(-r^{2} / 4 D t\right),
$$

where $c(r, t)$ is the concentration of the chemical, $A$ is a constant, $D$ is the diffusion coefficient, $t$ is the time after ejection, and $r$ is the distance from the source. The values $C(r, t)$ at various times after the onset of multiple ejections can be computed by summing the contribution from each pulse, namely,

$$
C(r, t)=\sum_{j=0}^{j_{o}} \frac{A \exp \left[-r^{2} / 4 D\left(t-j t_{o}\right)\right]}{4\left[\pi D\left(t-j t_{o}\right)\right]^{3 / 2}}
$$

where $j_{o}$ is the largest integer that is smaller than $t / t_{o}$ and $t_{o}$ is the inverse of frequency in seconds. The concentration gradient of the chemical can be obtained as $\partial C(r, t) / \partial r$.

Theoretical curves shown in Figure $2 b-d$ were constructed for the experimental conditions of a $20 \mathrm{~mm}$ pipette solution, single ejection volume of $0.3 \mathrm{pl}$, and $D$ value of $5.2 \times 10^{-6} \mathrm{~cm}^{2} / \mathrm{sec}$. These computations assume that the culture bath serves as an infinite sink to which the total amount of the ejected chemicals resulted in insignificant changes in the total bath concentration of the chemical. This assumption is reasonable, since the volume of the culture bath was $3 \mathrm{ml}$ and the total volume of the ejected solution under the higher frequency of application was only $4.4 \mathrm{nl}$ after $2 \mathrm{hr}$. For $20 \mathrm{~mm}$ solution ejected from the pipette, the final bath concentration of the chemical would be elevated to about $29 \mathrm{nM}$, a value much smaller than that produced near the growth cone at steady state $(30 \mu \mathrm{M}$ at $2 \mathrm{~Hz}$; Fig. $2 c)$. Finally, it may be noted that a $100 \mu \mathrm{m}$ distance from the growth cone was chosen for the standard ejection site since at this distance the steady-state value of the gradient is more significant than its rippling fluctuation. However, whether a time-varying gradient is as effective as the steady-state gradient in causing growth cone turning is unknown.

Microscopy and fluorescence measurement. Most of the experiments were carried out on an inverted phase-contrast microscope (Nikon Diaphot) with a $20 \times$ objective. The images were collected with a video camera (RCA CCD) and recorded on a videotape recorder (Panasonic AG 6300). Several experiments used a microscope equipped with highresolution DIC optics and a $40 \times$ oil-immersion objective (NA 0.9). The DIC image was collected by a CCD camera (Photometrics Star I), processed by an image processor (Imaging Technology 151), and stored on an optical memory disk recorder (Panasonic TQ 2026F). Fluorescence measurement was used to evaluate whether a diffusional process accounts for the spread of ejected chemicals from the site of ejection in a typical experiment. The fluorescence measured from the medium surrounding the micropipette at various times after the application of a pulse of fluorescein-dextran solution was recorded, using the system described above for DIC microscopy. The focal plane of the images was set at the surface of the culture substratum where the opening of the pipette was positioned. The intensity of a $6 \times 8$ pixel domain (covering a circle of $7.8 \mu \mathrm{m}^{2}$ ) was determined at various distances from the pipette 

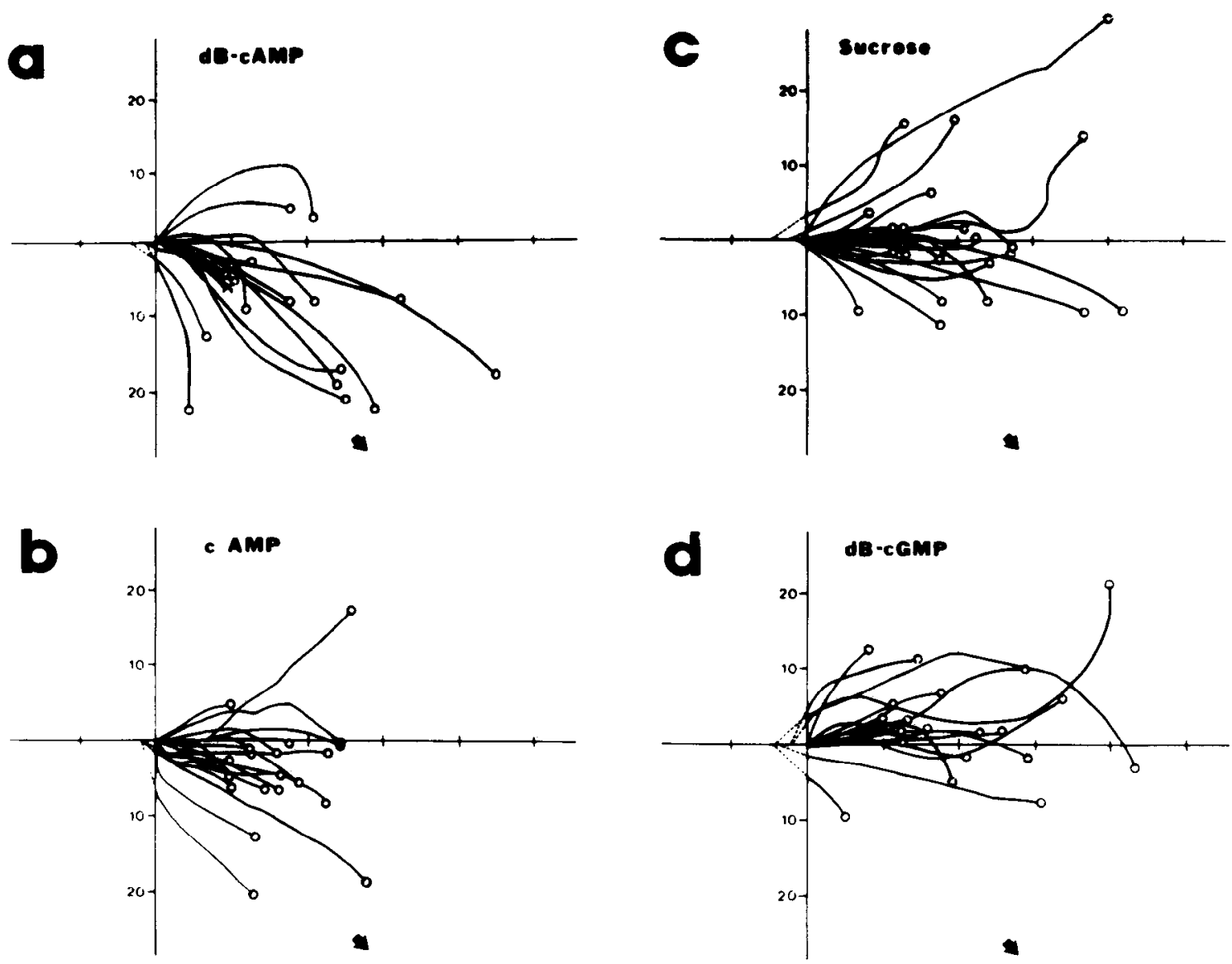

Figure 4. Composite drawings of neurites growing in cyclic nucleotide gradients. The graphs were made by superimposing tracings of video records of the microscopic images obtained at the end of the observation period for all neurites examined in a particular chemical gradient. The origin represents the position of the center of growth, which is defined as the center of the phase-dark palm of the growth cone, before the application of the gradient. The original direction of the neurite extension, as defined by the last $20 \mu \mathrm{m}$ segment of the neurite at the onset of the gradient, was aligned with the $\mathrm{x}$-axis. The line drawings depict the form of the neurite after $2 \mathrm{hr}$ of growth in the chemical gradient. Arrows point to the radial direction of chemical gradients, which were produced by pulsatile application of dB-cAMP $(a)$, native cAMP $(b)$, sucrose $(c)$, and dB-cGMP $(d)$. Note that some neurites had retracted a short distance from the origin before subsequent extension (broken lines). Tick marks along $\mathrm{x}$ - and $\mathrm{y}$-axes represent $10 \mu \mathrm{m}$.

tip (opening, $1 \mu \mathrm{m}$ ) for three different times after the pulse application. To reduce errors caused by experimental fluctuation, the intensity for each data point was obtained by averaging five independent measurements.

\section{Results}

\section{Analysis of the microscopic gradient}

In the present study, we used microscopic gradients generated by repetitive pulsatile ejection of picoliters of chemical solution near the growth cone. Our aim was to generate a stable gradient over a distance of tens of microns within several minutes. Figure 1 shows the appearance of a pulse of concentrated blue dye solution ejected from a pipette at various times $(0.067,0.3,1$, and $2 \mathrm{sec}$ ) during one cycle of pulsatile ejection at $0.5 \mathrm{~Hz}$. The distribution of the ejected dye with time is clearly determined by diffusion. To assay quantitatively the concentration gradient of the ejected substances, we measured the diffusion profile of fluorescein-conjugated dextran after a single ejection of the dextran solution from the micropipette. Figure $2 a$ depicts the fluorescence intensity at different distances from the ejection site at three different times after the ejection. The measured dye concentration with distance (data points) fits well with the diffusion profile (solid lines) predicted by theory (see Materials and Methods).
The properties of chemical gradients produced by repetitive ejection were analyzed for the pulse parameters used in experiments. Figure $2 b$ illustrates the predicted gradients produced at three distances from a $20 \mathrm{~mm}$ pulsatile source of a chemical with diffusion coefficient of $5.2 \times 10^{-6} \mathrm{~cm}^{2} / \mathrm{sec}$. We found that the gradient rapidly attained a stable pattern and that its characteristics at a distance of $100 \mu \mathrm{m}$ were most appropriate for our experiments. Assuming the bath serves as an infinite sink, the predicted concentration of the chemical near the growth cone builds up to a stable level within minutes. At fixed distance from the source, both the average concentration and the steepness of the gradient are increased by increasing pulse frequency (Fig. 2c,d).

\section{Effects of microscopic gradients on growth cone turning}

dB-cAMP gradient. Embryonic spinal neurons were prepared from $1 \mathrm{~d}$ Xenopus embryos. Within $6 \mathrm{hr}$ after plating, many dissociated neurons initiate active neurite outgrowth. Experiments were carried out at room temperature $6-12 \mathrm{hr}$ after plating, on neurons whose growing neurites did not contact other cells in the culture. A gradient of dB-cAMP was produced by repetitive ejection of picoliter volumes of $20 \mathrm{~mm}$ solution from a micropipette $100 \mu \mathrm{m}$ from the growth cone and $45^{\circ}$ from the direction of neurite extension. Video recordings of the neurons 
were made before and $2 \mathrm{hr}$ after the initiation of the gradient. Figure 3 illustrates the responses of growth cones in gradients of dB-cAMP and sucrose. Turning toward the source of dBcAMP is apparent, but no significant turning was observed when the same concentration of sucrose replaced dB-cAMP. The final patterns of neurite processes after the $2 \mathrm{hr}$ period were traced from the recorded video images for all growth cones that showed net extension. Figure 4 shows composite graphs of these tracings. The specific chemotactic action of dB-cAMP is clear; sucrose, underivatized CAMP, and $\mathrm{dB}-\mathrm{cGMP}$ of the same concentration all produced insignificant responses. The fact that cAMP did not produce significant turning suggests that $\mathrm{dB}$-cAMP does not produce its effect by binding to cell surface receptors. The slight effect of cAMP (Table 1) may be accounted for by intracellular action, due to its small membrane permeability.

To quantify the turning response and the net neurite extension, we determined the final position of the center of the growth cone at the end of the $2 \mathrm{hr}$ period in polar coordinates, using the growth cone position at the onset of the experiment as the origin. The results are shown in Table 1 . Note that if all neurites showed the ideal turning response the average angle of turning would be $45^{\circ}$. The differences between the average angle for $\mathrm{dB}$ cAMP and that for sucrose, cAMP, or dB-cGMP (at $20 \mathrm{~mm}$ ) were all statistically significant at $p<0.05$ (ANOVA and post hoc comparisons, and Mann-Whitney $U$ test), while the differences between that for sucrose and for cAMP and dB-cGMP were not significant $(p>0.05)$. Lowering the dB-cAMP concentration to $2 \mathrm{mM}$ reduced the turning response to an insignificant level, while raising the concentration to $200 \mathrm{~mm}$ or the pulse frequency to $1 \mathrm{~Hz}$ did not further increase the response, suggesting that $20 \mathrm{~mm}$ dB-cAMP had yielded near maximal effect. The percentage of neurites that grew during the observation period and the net neurite extension in various gradients were relatively constant; no significant differences wcre found between sucrose and all other treatments shown in Table $1(p$ $>0.05)$. Thus, at the level of dB-cAMP used in these gradient experiments, growth cone turning was initiated without significantly changing the growth rate.

Analysis of the chemical gradient produced by repetitive pulsatile applications indicates that, for experiments shown in Figures 3 and 4, the effective dB-cAMP gradient was about $0.1 \mu \mathrm{M}$ / $\mu \mathrm{m}$, or $1 \mu \mathrm{M}$ difference extracellularly across a typical growth cone $10 \mu \mathrm{m}$ in width. Since the average concentration of dBcAMP at $100 \mu \mathrm{m}$ from the source was between 8 and $9 \mu \mathrm{M}$ at the steady state, the growth cone was capable of responding to an approximate $10 \%$ difference in the concentration of external dB-cAMP across the growth cone. The cytoplasmic concentration of $\mathrm{dB}$-cAMP, however, is unknown.

$I B M X$ and forskolin gradients. The role of endogenous cAMP in the turning response of the growth cone was examined using drugs that alter the degradation or the production of cytosolic cAMP. We first tested the effect of a membrane-permeable phosphodiesterase inhibitor, IBMX. When a gradient of IBMX was produced by pulsatile application $(2 \mathrm{~Hz})$ of IBMX solution $(1.5$ $\mathrm{mM}$ ) at the same position as described for Figures 3 and 4, significant turning of the growth cone toward the source of IBMX was observed (see Table 1). A lower concentration of IBMX $(0.5 \mathrm{~mm})$ was not effective.

We also tested the effects of adenylate cyclase activation using a water-soluble derivative of forskolin (Laurenza et al., 1987). A gradient of this forskolin derivative was produced as described above, except that a slightly larger ejection volume was used. A marked turning response of the growth cone toward the source

\begin{tabular}{|c|c|c|c|}
\hline Gradient & $\begin{array}{l}\text { Turning } \\
\text { response } \\
\text { (degrees) }\end{array}$ & $\begin{array}{l}\text { Neurite } \\
\text { extension } \\
(\mu \mathrm{m})\end{array}$ & $N$ \\
\hline \multicolumn{4}{|l|}{ dB-cAMP } \\
\hline$(20 \mathrm{~mm}, 0.5 \mathrm{~Hz})$ & $29.1 \pm 4.9^{*}$ & $19.1 \pm 2.2$ & 20 \\
\hline \multicolumn{4}{|l|}{ Sucrose } \\
\hline$(20 \mathrm{~mm}, 0.5 \mathrm{~Hz})$ & $0.05 \pm 5.5$ & $21.3 \pm 2.3$ & 22 \\
\hline \multicolumn{4}{|l|}{ dB-cGMP } \\
\hline$(20 \mathrm{~mm}, 0.5 \mathrm{~Hz})$ & $-7.7+5.4$ & $20.4+2.2$ & 20 \\
\hline \multicolumn{4}{|l|}{ cAMP } \\
\hline$(20 \mathrm{~mm}, 0.5 \mathrm{~Hz})$ & $13.7 \pm 5.0$ & $17.7 \pm 1.4$ & 20 \\
\hline \multicolumn{4}{|l|}{ dB-cAMP } \\
\hline$(2 \mathrm{~mm}, 0.5 \mathrm{~Hz})$ & $10.6 \pm 9.9$ & $20.4 \pm 2.7$ & 10 \\
\hline \multicolumn{4}{|l|}{ dB-cAMP } \\
\hline$(200 \mathrm{~mm}, 0.5 \mathrm{~Hz})$ & $26.4 \pm 4.6^{*}$ & $27.3 \pm 4.2$ & 17 \\
\hline \multicolumn{4}{|l|}{ dB-cAMP } \\
\hline$(20 \mathrm{~mm}, 1 \mathrm{~Hz})$ & $27.7 \pm 5.1^{*}$ & $23.4 \pm 3.6$ & 10 \\
\hline \multicolumn{4}{|l|}{ IBMX } \\
\hline$(1.5 \mathrm{~mm}, 2 \mathrm{~Hz})$ & $22.5 \pm 7.8^{*}$ & $18.5 \pm 2.0$ & 10 \\
\hline \multicolumn{4}{|l|}{ IBMX } \\
\hline$(0.5 \mathrm{~mm}, 2 \mathrm{~Hz})$ & $9.3 \pm 7.7$ & $17.0 \pm 2.0$ & 15 \\
\hline
\end{tabular}

Gradients were produced by pulsatile application of picoliter volumes from a pipette positioned $100 \mu \mathrm{m}$ from the growth cone and $45^{\circ}$ from the direction of growth. All pulses were $4 \mathrm{msec}$ in duration. Concentration and frequency refer to the pipette concentration of the test substance and the pulse frequency.

* Significantly different from sucrose control group $(p<0.05$; ANOVA and post hoc comparisons, and Mann-Whitney $U$ test).

of forskolin (pipette concentration, $5 \mathrm{~mm}$ ) was apparent within $30 \mathrm{~min}$ after the onset of the gradient. Figure 5 shows composite drawings of neurites after $30 \mathrm{~min}$ and $1 \mathrm{hr}$ in the presence of a forskolin or sucrose gradient. Results of quantitative measurements of the turning angle are shown in Table 2.

It should be noted that neurite growth in these Xenopus cultures is episodic and that the average rate of extension usually shows gradual reduction during the initial phase of outgrowth. This may account for differences in rate of neurite extension during control experiments of different durations (Tables 1, 2). About $40-60 \%$ of the neurons examined in these studies did not show detectable growth during the observation period, and analysis of the turning response was only carried out for neurites that extended.

\section{Effects of forskolin and $d B-c A M P$ on neurite growth rate}

Application of a forskolin gradient in the above experiments also significantly increased the rate of neurite extension (see Table 2) and the percentage of neurites that extended ( $62 \%$ of cells exposed to a forskolin gradient compared to $41 \%$ of control cells). The increase in growth rate produced by forskolin was further investigated by acute bath application. The rate of neurite extension over a $2 \mathrm{hr}$ period was measured from time lapse video recordings of 24-30 individual neurites in each culture. The average growth rate in cultures treated with $10 \mu \mathrm{M}$ forskolin was $156 \pm 14 \%( \pm \mathrm{SEM} ; N=5)$ of that in parallel control cultures. The increase in growth rate was not observed when the forskolin concentration in the bath was lowered to $4 \mu \mathrm{M}$ (average rate, $106 \pm 7 \%$ of the control, $\pm \mathrm{SEM} ; N=5$ cultures). Theoretical calculations similar to those shown in Figure 2 indicate that the average forskolin concentration near the growth cone, with pulse parameters as those described in Figure 5, was about 10-20 $\mu \mathrm{M}$. Thus, the increased growth rate observed in 

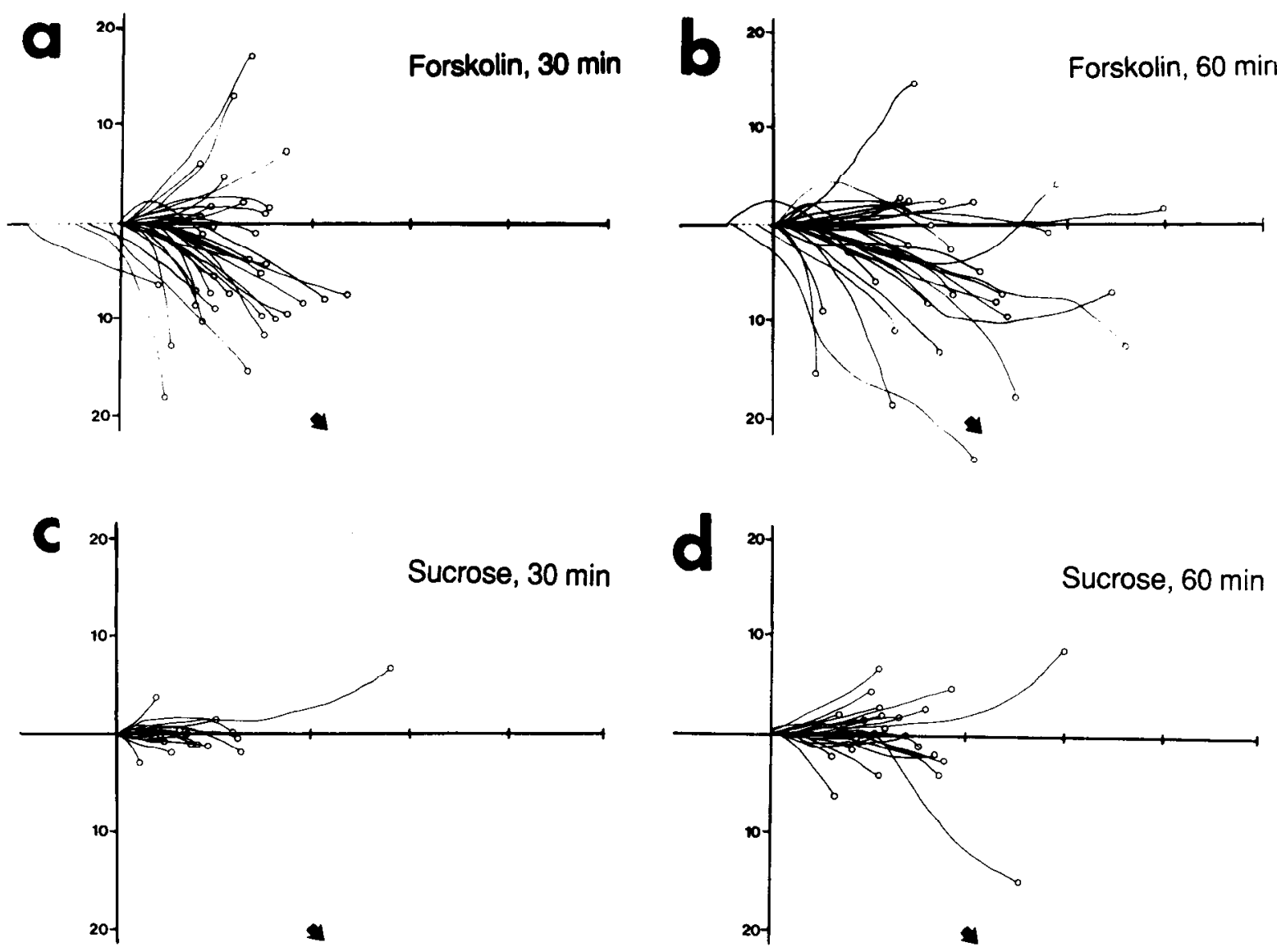

Figure 5. Effect of forskolin gradient. Composite drawings (as described in Fig. 4 ) of neurites in a gradient of forskolin $(a, b)$ or sucrose $(c, d)$, for $30 \mathrm{~min}$ and $1 \mathrm{hr}$, respectively. The gradient was applied in the same manner as that described in Figure 3, except that the frequency and duration of the pulses were $2 \mathrm{~Hz}$ and $10 \mathrm{msec}$, and the ejection volume averaged $0.65 \mathrm{pl}$. Tick marks along $\mathrm{x}$ - and $\mathrm{y}$-axes represent $10 \mu \mathrm{m}$.

the forskolin gradient experiments is consistent with the above observation on the promotion of neurite growth by uniform bath application of forskolin.

As described earlier, we did not observe a significant change in the neurite growth rate in the $\mathrm{dB}$-cAMP gradient experiment. A more detailed study was carried out on neurite growth in cultures supplemented with different concentrations of dBcAMP. The average growth rate of neurites in cultures containing $20 \mu \mathrm{M} \mathrm{dB}-\mathrm{cAMP}$ was $19.4 \pm 1.1 \mu \mathrm{m} / \mathrm{hr}( \pm \mathrm{SEM} ; N=4$ cultures), not significantly different from the growth rate in control cultures $(18.4 \pm 1.7 \mu \mathrm{m} / \mathrm{hr} ; N=4)$. At a much higher $\mathrm{dB}$ cAMP concentration ( $2 \mathrm{mM}$ ), the growth rate was significantly increased $(25.44 \pm 2.6 \mu \mathrm{m} / \mathrm{hr} ; N=4 ; p<0.05, t$ test $)$. The average concentration at the growth cone reached only $8-80 \mu \mathrm{M}$ during the dB-cAMP turning experiments (depending on pipette concentration and pulse frequency; see Table 1, Fig. 2c), not sufficient to increase the growth rate. We thus conclude that $\mathrm{dB}$ cAMP can increase the rate of neurite extension but that this increase is not required for the turning response.

\section{Growth cone behavior during the turning response}

In order to observe in more detail the cellular events underlying the turning response, the behavior of the growth cone in a forskolin gradient was monitored with video-enhanced DIC microscopy at high resolution. Figure $6 a-h$ shows two turning responses by the same cell in an experiment where the forskolin gradient was applied first from the right and then (after the growth cone had turned) from the left. The orientation responsc was detectable in this case within 5-10 min, when little neurite extension had occurred. The response of the growth cone of another neuron is shown in Figure $6 i-l$. In this case the rate of extension was slower, and the time elapsed before orientation occurred was much longer ( $\sim 40 \mathrm{~min})$.

From some of the recorded video images, the number and the length of filopodia on both sides of the growth cone (with respect to the direction of extension) were measured over a period of 5-10 min before visible turning of the central region of the growth cone. Counts of filopodial number and total filopodial length on the sides toward and opposite the pipette were averaged for three to eight observations of each growth cone in the minutes preceding visible turning, and the normalized values from four separate turning events were calculated. The side of the growth cone facing the pipette (toward which the growth cone turned a few minutes later) supported an average of $51.1 \%$ of the total filopodial length and $50.4 \%$ of the total filopodial number ( $N=4$ turning events). Neither measure represented a significant asymmetry, suggesting that a preferential increase in the number or length of filopodia on one side of the growth cone may not be required for growth cone turning produced by the forskolin gradient. Although directed protrusion of the central portion of the growth cone need not be preceded by a visible difference in the number or length of filopodia, the possible 
Table 2. Response of growth cones in forskolin gradient

\begin{tabular}{lccc} 
& $\begin{array}{l}\text { Turning } \\
\text { response } \\
\text { Gegrees })\end{array}$ & $\begin{array}{l}\text { Neurite } \\
\text { extension } \\
(\mu \mathrm{m})\end{array}$ & $N$ \\
\hline Forskolin $(30 \mathrm{~min})$ & $19.9 \pm 5.4^{*}$ & $14.5 \pm 0.8^{*}$ & 34 \\
Sucrose $(30 \mathrm{~min})$ & $-3.7 \pm 4.4$ & $8.6 \pm 1.5$ & 16 \\
Forskolin $(60 \mathrm{~min})$ & $18.0 \pm 6.0^{*}$ & $21.0 \pm 1.5^{*}$ & 29 \\
Sucrose $(60 \mathrm{~min})$ & $-1.7 \pm 4.1$ & $12.9 \pm 1.2$ & 26 \\
\hline
\end{tabular}

Forskolin, $7 \beta$-desacetyl-7 $\beta$-[ $\gamma$-( $N$-methylpiperazino)-butyryl]. The gradient was produced by repetitive application of picoliter volumes of a $5 \mathrm{~mm}$ solution $(2 \mathrm{~Hz}$, $10 \mathrm{msec}$ ), $100 \mu \mathrm{m}$ from the growth cone and $45^{\circ}$ from the initial direction of neurite outgrowth.

* Significantly different from sucrose control group (Mann-Whitney $U$ test and Student's $t$ test, $p<0.02$ ).
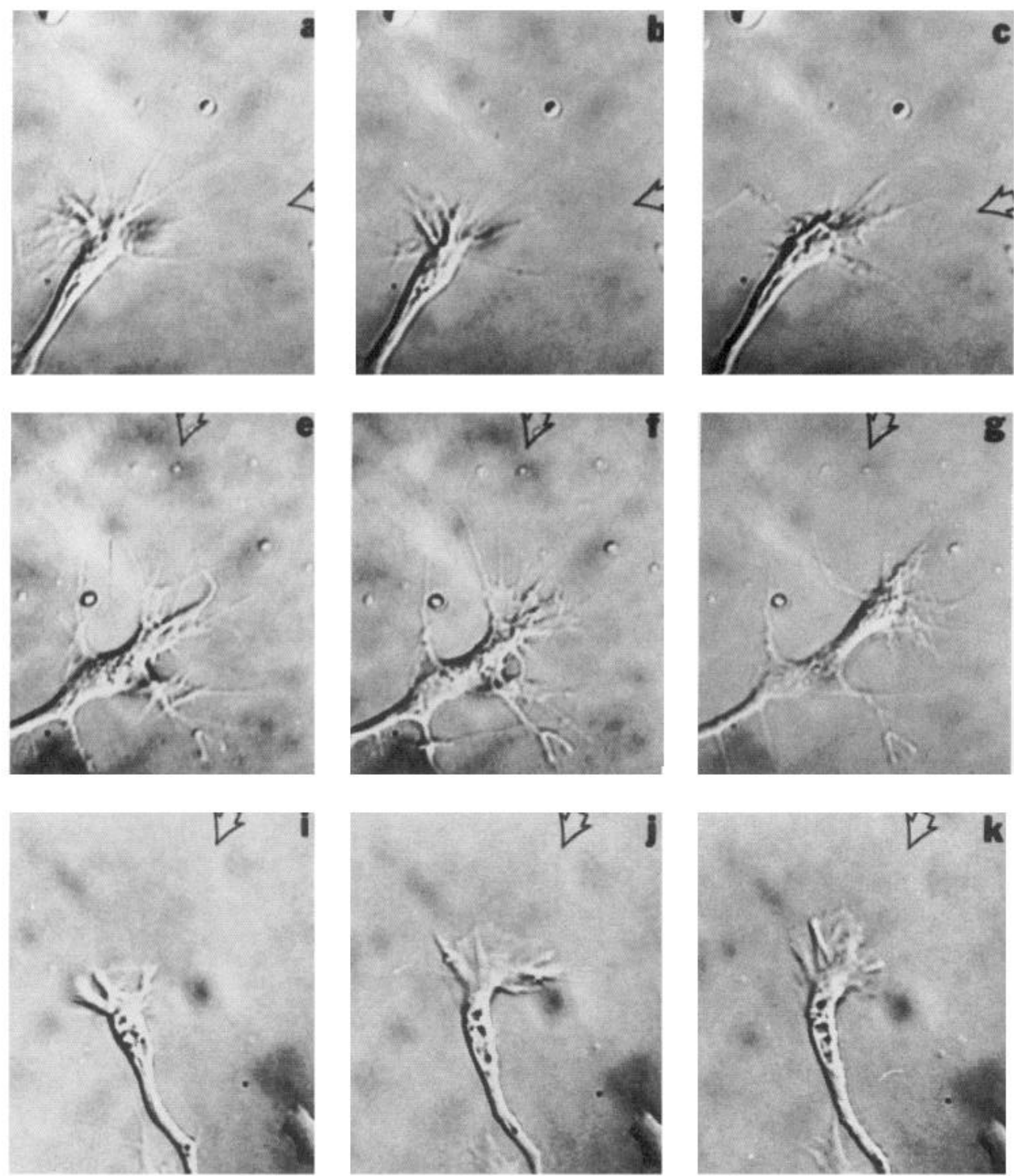
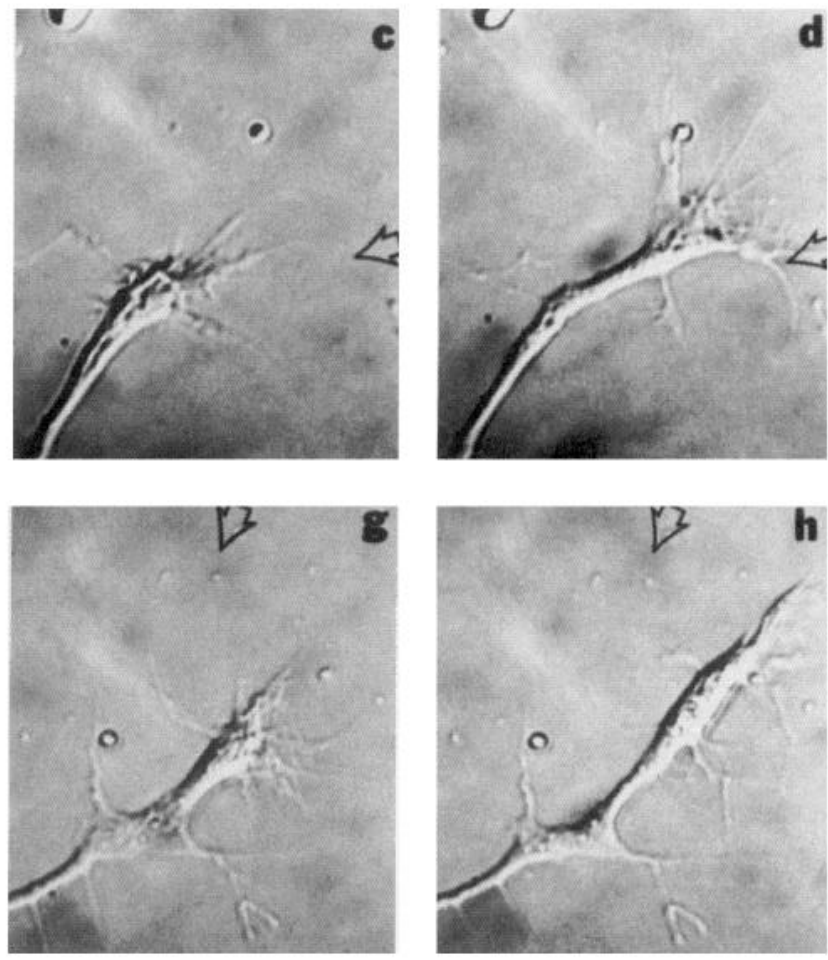

During the development of the nervous system, the growth cone encounters a complex environment within the developing tissue. A variety of physical and chemical factors are likely to take part in influencing the direction of nerve growth. In this work, we tested the hypothesis that an asymmetric distribution of cytoplasmic cAMP may be capable of mediating signals from the extracellular environment to induce the turning response of the growth cone. It is reasonable to expect that, despite the diversity of extracellular factors and neuronal types, the same cytoplasmic processes may be required for the turning response in many cases. For example, phosphorylation of certain cytoskeletal or regulatory proteins by second messenger-dependent protein kinases could serve as a common mediator for the action of many

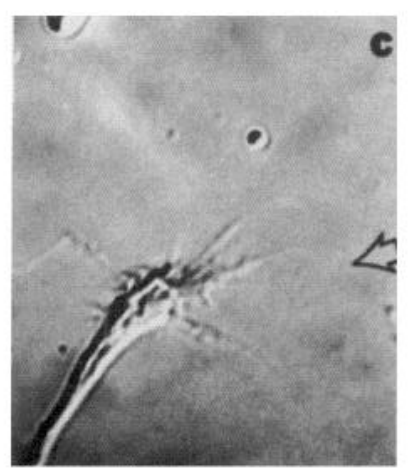

d

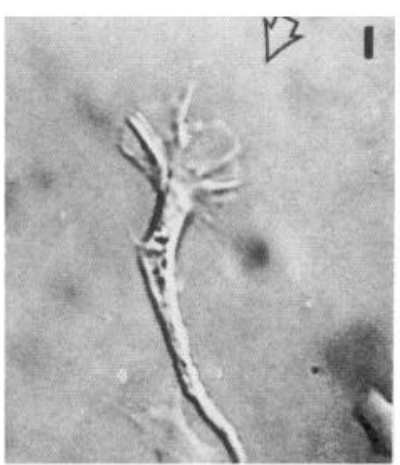

Figure 6. Morphology of the growth cone in the presence of forskolin gradient. $a-d$, Micrographs of a growth cone 0:00, 1:20, 5:20, and 24:26 $\mathrm{min}$ after the onset of the forskolin gradient, produced as described in Figure 5. The arrows indicate the direction of the gradient; the source of forskolin was $100 \mu \mathrm{m}$ away (out of the field). $e-h$, Micrographs of the same growth cone 5:10, 9:10, 25:10, and 47:54 min after the forskolin pipette had been repositioned to the left side of the growth cone. $i-l$, Micrographs of a different neuron with a slower growth rate 20:00, 41:00, 45:00, and $62: 20 \mathrm{~min}$ after the onset of a forskolin gradient applied from the right. 
extracellular cues. Results from the present study indicate that cAMP could serve as such a second messenger. In this light, it is interesting to note that cAMP elevation has been shown to mimic or potentiate some of the effects of NGF such as changes in membrane characteristics, protein phosphorylations, and ncurite outgrowth in PC12 cells (Heidemann et al., 1985; McTigue et al., 1985; Richter-Landsberg and Jastorff, 1986; Sano et al., 1990) and some NGF-responsive neurons (Rydel and Greene, 1988), although cAMP probably does not mediate the effect of NGF.

Individual cells were responsive to the applied gradients to different degrees. This Xenopus culture consists of a heterogeneous population of neurons, and the difference in the growth cone response may reflect intrinsic differences between neuronal types. Alternatively, all growth cones may be affected by the cAMP gradient but have different intrinsic directional biases prior to the experiment. The imposed cAMP gradient may be insufficient to alter substantially the direction of growth for those neurites that had been influenced toward a diametrically different direction by some unknown factor in the culture environment. For example, prior to the application of the gradient, a growth cone may have become committed to alter its direction of extension but not yet expressed the morphological change in orientation. It is interesting to note that a similar range of average turning angles was also found in these cultures for the turning induced by a very different external factor, namely, a focal electric field (Patel and Poo, 1984).

Isolated rat brain growth cones contain cAMP-dependent phosphorylation (Ellis et al., 1985) and adenylate cyclase activity responsive to external stimuli (Lockerbie et al., 1988). In addition, some microtubule-associated proteins (MAPs) have been localized in growth cones (Brugg and Matus, 1988; Sano et al., 1990). In vitro studies have shown that phosphorylation of MAPs reduces their affinity for tubulin and the rate of $\mathrm{mi}$ crotubule assembly (Jameson and Caplow, 1981; Murthy and Flavin, 1983; Lindwall and Cole, 1984) and inhibits the crosslinking of actin filaments by MAPs (Nishida et al., 1981; Selden and Pollard, 1983; Sattilaro, 1986). Since microtubule polymerization and cytoskeletal interactions are essential for directed neurite outgrowth, local modulation of these processes through cAMP-dependent phosphorylation at the growth cone could regulate the direction of neurite extension.

Chemotaxis in response to external signals has been previously reported. Chick dorsal root ganglion nerve growth cones orient and grow toward a source of NGF (Letourneau, 1978; Gunderson and Barrett, 1979, 1980), and leukocytes show chemotaxis in response to certain peptides (Carson et al., 1986). In the latter case, it appears that binding of chemotactic peptides to cell surface receptors locally increases cytoplasmic actin nucleation and polymerization. In the present study, we did not observe increased filopodial length or number on the side of the growth cone facing the source of forskolin prior to turning of its central region, suggesting that preferential growth of filopodia docs not mediate the forskolin-induced turning of Xenopus growth cones. Moreover, the symmetry of filopodia number around the central region of the growth cone appeared to be maintained during the turn (Fig. 6). These observations suggest that an asymmetric distribution of filopodia is unlikely to be the primary means by which the forskolin gradient produces turning. Differential rates of microtubule polymerization or stabilization, asymmetric changes in actin cytoskeleton, or the selective translocation and insertion of membrane precursor ves- icles on one side of the growth cone are all possible alternative mechanisms. The full turning response of the growth cone must involve asymmetric membrane addition as well as cytoskeletal reorganization, but the exact sequence of events and causal relationships among them remain to be elucidated.

In conclusion, we have demonstrated a simple and effective method of producing microscopic chemical gradients for testing chemotactic behavior of growing nerve processes. While the primary extracellular signal for growth cone turning may not necessarily be a diffusible chemotactic agent, a cAMP gradient within the growth cone cytoplasm could serve as a common second messenger that couples some extracellular cues to the cytoplasmic processes responsible for the turning response.

\section{References}

Anderson MJ, Cohen MW, Zorychta E (1977) Effects of innervation on the distribution of acetylcholine receptors on cultured muscle cells. J Physiol (Lond) 268:731-756.

Bixby JL (1989) Protein kinase $C$ is involved in laminin stimulation of neurite outgrowth. Neuron 3:287-297.

Bray D, Hollenbeck PJ (1988) Growth cone motility and guidance. Annu Rev Cell Biol 4:43-61.

Brugg B, Matus A (1988) PC12 cells express juvenile microtubuleassociated proteins during nerve growth factor-induced neurite outgrowth. J Cell Biol 107:643-650.

Carson M, Weber A, Zigmond SH (1986) An actin-nucleating activity in polymorphonuclear leukocytes is modulated by chemotactic peptides. J Cell Biol 103:2707-2714

Ellis L, Katz F, Pfenninger KH (1985) Nerve growth cones from fetal rat brain. II. Cyclic adenosine $3^{\prime}, 5^{\prime}$-monophosphate (cAMP)-binding proteins and cAMP-dependent phosphorylation. J Neurosci 5:13931401.

Forscher P, Smith SJ (1988) Actions of cytochalasins on the organization of actin filaments and microtubules in a neuronal growth cone. J Cell Biol 107:1505-1516.

Forscher P, Kaczmarek LK, Buchanan J, Smith SJ (1987) Cyclic AMP induces changes in distribution and transport of organelles within growth cones of Aplysia bag cell neurons. J Neurosci 7:3600-3612.

Greene LA, Drexler AA, Connoly JL, Rukenstein A, Green SH (1986) Selective inhibition of responses to nerve growth factor and microtubule-associated protein phosphorylation by activation of adenylate cyclase. J Cell Biol 103:1967-1978.

Gunderson RW, Barrett JN (1979) Neuronal chemotaxis: chick dorsalroot axons turn toward high concentrations of nerve growth factor. Science 206:1079-1080.

Gunderson RW, Barrett JN (1980) Characterization of the turning response of dorsal root neurites toward nerve growth factor. J Cell Biol 87:546-554.

Heidemann SR, Joshi HC, Schechter A, Fletcher JR, Bothwell M (1985) Synergistic effects of cyclic AMP and nerve growth factor on neurite outgrowth and microtubule stability of PC12 cells. J Cell Biol 100: 916-927.

Jameson L, Caplow M (1981) Modification of microtubule steadystate dynamics by phosphorylation of the microtubule-associated proteins. Proc Natl Acad Sci USA 78:3413-3417.

Lankford KL, DeMello FG, Klein WL (1988) D1-type dopamine receptors inhibit growth cone motility in cultured retina neurons: evidence that neurotransmitters act as morphogenic growth regulators in the developing central nervous system. Proc Natl Acad Sci USA $85: 2839-2843$.

Laurenza A, Khandelwal Y, De Souza NJ, Rupp RH, Metzger H, Seamon KB (1987) Stimulation of adenylate cyclase by water-soluble analogues of forskolin. Mol Pharmacol 32:133-139.

Letourneau PC (1978) Chemotactic response of nerve fiber elongation to nerve growth factor. Dev Biol 66:183-196.

Letourneau PC (1985) Axonal growth and guidance. In: Molecular bases of neural development (Edelman GM, Gall WE, Cowan WM, eds), pp 269-293. New York: Wiley.

Lindwall G, Cole RD (1984) Phosphorylation affects the ability of tau protein to promote microtubule assembly. J Biol Chem 259:53015305.

Lockerbie RO, Herve D, Blanc G, Tassin J-P, Glowinski J (1988) 
Isolated neuronal growth cones from developing rat forebrain possess adenylate cyclase activity which can be augmented by various receptor agonists. Dev Brain Res 38:19-25.

Mattson MP, Taylor-Hunter A, Kater SB (1988) Neurite outgrowth in individual neurons of a neuronal population is differentially regulated by calcium and cyclic AMP. J Neurosci 8:1704-1711.

McTigue M, Cremins J, Halegoua S (1985) Nerve growth factor and other agents mediate phosphorylation and activation of tyrosine hydroxylase. J Biol Chem 260:9047-9056.

Murthy ASN, Flavin M (1983) Microtubule assembly using the microtubule-associated protein MAP2 prepared in defined states of phosphorylation with protein kinase and phosphatase. Eur J Biochem 137:37-46

Nieuwkoop PD, Faber J (1967) Normal table of Xenopus laevis (Daudin), 2d ed. Amsterdam: North-Holland.

Nishida E, Kuwaki T, Sakai H (1981) Phosphorylation of microtubuleassociated proteins (MAPs) and $\mathrm{pH}$ of the medium control interaction between MAPs and actin filaments. J Biochem (Tokyo) 90:575-578.

Patel N, Poo M-m (1984) Perturbation of the direction of neurite growth by pulsed and focal electric fields. J Neurosci 4:2939-2947.

Richter-Landsberg C, Jastorff B (1986) The role of cAMP in nerve growth factor-promoted neurite outgrowth in $\mathrm{PCl} 2$ cells. J Cell Biol 102:821-829.

Rydel RE, Greene LA (1988) cAMP analogs promote survival and neurite outgrowth in cultures of rat sympathetic and sensory neurons independently of nerve growth factors. Proc Natl Acad Sci USA 85: $1257-1261$.

Sano M, Katoh-Semba R, Kitajima S, Sato C (1990) Changes in levels of microtubule-associated proteins in relation to the outgrowth of neurites from PC12D cells, a forskolin- and nerve growth factorresponsive subline of $\mathrm{PC} 12$ pheochromocytoma cells. Brain Res 510 : 269-276.

Sattilaro RF (1986) Interaction of microtubule-associated protein with actin filaments. Biochemistry 25:2003-2009.

Selden SC, Pollard TD (1983) Phosphorylation of microtubule-associated proteins regulates their interaction with actin filaments. $\mathrm{J}$ Biol Chem 258:7064-7071.

Spitzer NC, Lamborghini JC (1976) The development of the action potential mechanism of amphibian neurons isolated in culture. Proc Natl Acad Sci USA 73:1641-1645.

Tabti N, Poo M-m (1991) Culturing spinal neurons and muscle cells from Xenopus embryos. In: Culturing nerve cells (Banker G, Goslin K, eds). Cambridge, MA: MIT Press.

Tsuda M, Ono K, Katayama N, Yamagata Y, Kikuchi K, Tsuchiya T (1989) Neurite outgrowth from mouse neuroblastoma an cerebellar cells induced by the protein kinase inhibitor H-7. Neurosci Lett 105: 241-245.

Weast RC (1984) Handbook of chemistry and physics, 65th ed, p F-45. Boca Raton, FL: CRC. 